\title{
ANISOTROPY OF THE SURFACE OF CUBIC BODY-CENTERED CRYSTAL LATTICES
}

\author{
Yurov V.M. ${ }^{1}$, Goncharenko V.I. ${ }^{2}$, Oleshko V.S. ${ }^{2}$ \\ ${ }^{1}$ E.A. Buketov Karaganda University, Karaganda, Kazakhstan, exciton@list.ru \\ 2 Moscow Aviation Institute (National Research University), Moscow, Russia, ovs_mai@mail.ru
}

\begin{abstract}
In the work of Shebzukhova and Arefieva, by the method of electronic-statistical calculation of the anisotropy of the surface energy of metals, a method for estimating the work function of electrons from a metal was determined. The surface energy and electron work function of four main faces of cadmium and zinc crystals and five faces of mercury are estimated. In the work of Bokarev, the anisotropy of the surface energy of single crystals was calculated from the model of coordination melting of crystals. Based on experimental studies and theoretical calculations, it is shown that the model of coordination melting of crystals unambiguously links the physicochemical properties of the surface of single crystals with their crystal structure. In our proposed empirical model, not only the anisotropy is calculated, but also the thickness of the surface layer of the metal. It is shown that the thickness of the surface layer is determined by one fundamental parameter - the molar (atomic) volume, which periodically changes in accordance with the table of D.I. Mendeleev. It is shown in the work that the work function of electrons changes proportionally with a change in the surface energy of the metal. This means that the device we have developed can be used to measure the state of the metal surface and its anisotropy.
\end{abstract}

Keywords: anisotropy, metal, surface energy, crystal, nanostructure.

\section{Introduction}

This work is a continuation of works [1,2], where it is shown that for the operational measurement of the energy state of the metal surface, it is necessary to use non-destructive testing methods. The method of measuring the contact potential difference (or the work function of an electron from the surface) was chosen as such a control. Here the measuring electrode of the device, at which the contact potential difference and the investigated metal surface are measured, form a kind of capacitor, between which this contact potential difference is formed. The positive aspect of the capacitor method for measuring the contact potential difference is:

- high precision in capacitor manufacturing;

- the capacitor in its normal state has low heat losses and a high efficiency;

- electrical fields have little effect on the mechanical parts of the capacitor;

- the sensor of the device can be made by giving it the shape of the investigated surface.

The portable complex for measuring the work function of electrons developed by the authors on the basis of a portable digital oscilloscope makes it possible to perform non-destructive testing of the surface of metal parts in a continuous mode during their production, operation or repair.

We used this complex to determine the surface energy of the surface of pure metals and alloys that are used in aviation. This work is of a theoretical nature and is devoted to the surface of body-centered cubic crystal lattices.

\section{Analysis of publications}

First of all, we will note only those publications that concern the anisotropy of the metal surface, to which we will devote our presentation. A significant number of works have been devoted to the anisotropy of surface energy and the work function of electrons from metals, since it must be taken into account in solving practical problems faced by both technicians and technologists.

For example, in the microelectronic industry materials with design standards of $16-14 \mathrm{~nm}$ are already being used, and technologies with design standards at the level of $10^{-7} \mathrm{~nm}$ are being mastered. However, such a transition to the nanoscale requires a transition to work with new materials. In this case, the quantities of 
the above-mentioned regions determine their size dependence and the surface energy of the crystal and its anisotropy have a significant dependence on the properties of materials.

In works [3, 4], by the method of electron-statistical calculation of the surface energy of metals, a method was determined for the work function of electrons from a metal with structures having hexagonal and rhombic syngony. The relationship between the surface energy and the work function of the electron is obtained. These calculations were performed for crystals of zinc, cadmium, and mercury. The formula for the work function of an electron $\varphi(\mathrm{hkl})$ and surface energy $f_{\omega}(\mathrm{hkl})$ of close-packed faces of metallic macrocrystals with noncubic structures is as follows [3]:

$$
\varphi(h \mathrm{kl})=-\left(\frac{\partial \mathrm{E}_{0}}{\partial \mathrm{z}}\right)_{\mathrm{R}}-\frac{1}{\mathrm{n}_{v} \tau}\left(\frac{\partial \mathrm{f}_{w}(\mathrm{hkl})}{\partial \mathrm{z}}\right)_{\mathrm{R}} .
$$

Here, the first term is a certain constant, independent of the indices of the crystallographic faces, the second determines the orientational dependence of the work function of electrons, which can be determined using Thomas - Fermi expressions for the variation of the electron density at the metal - vacuum interface with corrections [4]:

$$
\mathrm{f}_{\text {w }}(\mathrm{hkl}) \cong \frac{1}{5} \mathrm{n}(\mathrm{hkl})\left|\mathrm{W}\left(\mathrm{r}_{0}\right)\right| \mathrm{Q} \text {. }
$$

In formulas (1) and (2), a is the crystal lattice parameter, $\mathrm{z}$ is the number of free electrons per atom, $\mathrm{Q}$ is a function that weakly depends on $\mathrm{z},\left|\mathrm{W}\left(\mathrm{r}_{0}\right)\right|$ is the bond energy of the crystal lattice, $\mathrm{n}(\mathrm{hkl})$ is the concentration of particles on the face. The summation in (2) for each face was carried out up to the $j$-th plane, at which the contribution to the surface energy was $\leq 0.1 \mathrm{~mJ} / \mathrm{m}^{2}$.

With the effective thickness of the monatomic surface layer $\tau=\mathrm{R}$ (where $\mathrm{R}$ is the radius of the s-sphere) and bearing in mind (2), we obtain [3, 4]:

$$
\varphi(\mathrm{hkl})+\frac{\mathrm{B}}{\mathrm{z}} \mathrm{a}^{2} \cdot \mathrm{f}_{w}(\mathrm{hkl})=\text { const. }
$$

The constant B depends on the type of lattice structure of the metal and in the first approximation for hcp crystals is 3.51 , and for crystalline mercury with a rhombohedral lattice 5.846. Expression (3) conveys well the anisotropy $\varphi(\mathrm{hkl})$ of metals. To estimate the anisotropy of the work function of electrons, formula (3) is reduced to the form $[3,4]$ :

$$
\varphi(\mathrm{hkl})=\varphi_{0}\left(1-\frac{\mathrm{f}_{\varpi}^{\mathrm{o}}(\mathrm{hkl})}{\mathrm{f}_{0}}\right) .
$$

Here $\varphi_{0}=$ const $=\varphi+B / z^{2} f_{\omega}, f_{0}=\varphi_{0} z / B_{2}, \varphi$ is the Richardson work function of electrons from the polycrystalline sample, $f_{\omega}$ is the surface tension of the liquid metal at the melting temperature $T_{m}, f_{\omega}(h k l)$ is the surface energy of the faces at a given temperature. Using expressions (2) and (4), the surface energy and work function of electronically four main faces of cadmium and zinc crystals and five faces of mercury were estimated. Thus, for zinc, the deviation of the calculated value of $\varphi(0001)$ at $293 \mathrm{~K}$ from $\varphi_{\exp }(0001)$ and from the value for a polycrystal is 7.94 and $7.59 \%$, respectively. And for cadmium, the calculation error in comparison with the work function of electrons of the polycrystal is only $5.3 \%$. In view of the absence in most cases and the ambiguity of the available experimental data on the surface energies of the faces of single crystals with noncubic structures, thin films and nanoparticles of the considered metals, expressions (3) and (4) can be used to estimate the surface energies of metal surfaces at the interface with vacuum from the work function data electrons of these surfaces.

In $[5,6]$, the relationship between the anisotropy of the surface energy of single crystals and the crystal structure is considered. Based on experimental studies and theoretical calculations, it is shown that the model of coordination melting of crystals (MCP) unambiguously links the physicochemical properties of the surface of single crystals with their crystal structure. The calculations of the surface energy will be carried out according to the formula derived under the assumption that there are no first-order phase transitions in these substances up to their melting point $[5,6]$ :

$$
\sigma_{\mathrm{hkl}}=\left(\frac{\rho \mathrm{l}_{\mathrm{hkl}}}{\mathrm{M}}\right) \int_{0}^{\mathrm{T}_{\mathrm{m}}} \mathrm{c}_{\mathrm{p}} \mathrm{dT} \text {. }
$$


Materials science. 11

where $\rho$ is the density of the crystalline substance, and $\mathrm{M}$ is its molecular weight, Tm is the melting point, $\mathrm{cp}$ is the molar heat capacity, [hkl] is the thickness of the first coordination sphere in the [hkl] direction, which for crystals with body-centered (bcc) and face-centered (fcc) ) cubic structure is given by the relations [6]:

$$
\begin{aligned}
& \operatorname{Im} 3 \mathrm{~m}, \mathrm{Z}=2 ; 1_{100}=\mathrm{a} ; 1_{110}=\mathrm{a} \sqrt{2} ; 1_{111}=\mathrm{a} / \sqrt{3}, \\
& \text { Fm3m, } Z=4 ; 1_{100}=\mathrm{a} ; 1_{110}-\mathrm{a} / \sqrt{2} ; 1_{111}=2 \mathrm{a} / \sqrt{3} .
\end{aligned}
$$

We take the values of $\rho, \mathrm{M}, \mathrm{Tm}$ and $\mathrm{cp}$ for metals from the reference book [7]. Table 1 shows the calculations of the surface energy anisotropy by the MCP in comparison with the calculations of the surface energy anisotropy and the electron work function by several theoretical models [2-4].

Table 1. Anisotropy of the surface energy of metals, calculated from the MCP in comparison with the anisotropy

\begin{tabular}{|c|c|c|c|c|c|c|c|c|}
\hline $\mathrm{Me}$ & (hkl) & Structure & $\begin{array}{c}\sigma_{\mathrm{hkl}} \\
\text { by MCP, } \\
\mathrm{mJ} / \mathrm{m}^{2}[6]\end{array}$ & $\begin{array}{c}\sigma_{\mathrm{hkl}} \\
{[10]} \\
\mathrm{mJ} / \mathrm{m}^{2}\end{array}$ & $\begin{array}{c}\sigma_{\mathrm{hkl}}[8], \\
\mathrm{mJ} / \mathrm{m}^{2}\end{array}$ & $\begin{array}{c}\mathrm{F}_{\mathrm{hkl}}, \mathrm{eV} \\
{[10]}\end{array}$ & $\mathrm{F}_{\mathrm{hkl}}, \mathrm{eV}[8]$ & $\mathrm{F}_{\mathrm{hkl}}, \mathrm{eV}$ [9] \\
\hline \multirow{3}{*}{$\mathrm{Li}$} & 100 & \multirow{3}{*}{$\operatorname{Im} 3 \mathrm{~m}$} & 304 & 436 & 541 & 3.15 & 2.968 & 2.28 \\
\hline & 110 & & 430 & 458 & 585 & 3.33 & 3.221 & 2.82 \\
\hline & 111 & & 180 & - & 601 & - & 2.746 & 2.11 \\
\hline \multirow{3}{*}{$\mathrm{Na}$} & 100 & \multirow{3}{*}{$\operatorname{Im} 3 \mathrm{~m}$} & 189 & 236 & 258 & 2.76 & 2.638 & 2.28 \\
\hline & 110 & & 267 & 307 & 247 & 2.94 & 2.839 & 2.82 \\
\hline & 111 & & 109 & - & 302 & - & 2.585 & 2.11 \\
\hline \multirow{3}{*}{ K } & 100 & \multirow{3}{*}{$\operatorname{Im} 3 \mathrm{~m}$} & 124 & 129 & 148 & 2.34 & 2.224 & 2.25 \\
\hline & 110 & & 175.3 & 116 & 137 & 2.38 & 2.372 & 2.79 \\
\hline & 111 & & 71.6 & 112 & 165 & 2.41 & 2.18 & 2.08 \\
\hline \multirow{3}{*}{$\mathrm{Rb}$} & 100 & \multirow{3}{*}{$\operatorname{Im} 3 \mathrm{~m}$} & 101 & 107 & 126 & 2.22 & 2.116 & 2.16 \\
\hline & 110 & & 143 & 92 & 110 & 2.32 & 2.243 & 2.68 \\
\hline & 111 & & 58.4 & 89 & 135 & 2.29 & 2.096 & 2.00 \\
\hline \multirow{3}{*}{ Cs } & 100 & \multirow{3}{*}{$\operatorname{Im} 3 \mathrm{~m}$} & 85 & 92 & 114 & 2.03 & 1.974 & 1.87 \\
\hline & 110 & & 120 & 72 & 97 & 2.09 & 2.073 & 2.34 \\
\hline & 111 & & 49 & 70 & 119 & 2.10 & 1.971 & 1.72 \\
\hline
\end{tabular}
of the surface energy and the work function of the electron, known from the literature

As can be seen from Table 2, the calculated values of the work function of different crystal faces are proportional to the surface energies of these faces, calculated using the model of coordination crystal melting. The main goal of this work is to build a model of the surface layer of perfect single crystals and to clarify the role of surface energy in physical processes occurring in the nanoscale range.

Table 2. Anisotropy of the surface energy of metals calculated by (13) in comparison with the anisotropy of the

\begin{tabular}{|c|c|c|c|c|c|c|c|c|}
\hline$\overline{\mathrm{Me}}$ & (hkl) & Structure & $\mathrm{T}_{\mathrm{m}}, \mathrm{K}$ & $\mathrm{R}(\mathrm{I}), \mathrm{nm}$ & $\begin{array}{c}\sigma_{\mathrm{hkl}} \\
\text { by }(13), \mathrm{mJ} / \mathrm{m}^{2}\end{array}$ & $\begin{array}{c}\sigma_{\mathrm{hkl}} \text { by MCP, } \\
\mathrm{mJ} / \mathrm{m}^{2}[6]\end{array}$ & $\begin{array}{c}\sigma_{\mathrm{hkl}} \\
{[10], \mathrm{mJ} / \mathrm{m}^{2}}\end{array}$ & $\begin{array}{c}\mathrm{F}_{\mathrm{hkl}}, \mathrm{eV} \\
{[8]}\end{array}$ \\
\hline \multirow{3}{*}{$\mathrm{Li}$} & 100 & \multirow{3}{*}{$\operatorname{Im} 3 \mathrm{~m}$} & \multirow{3}{*}{454} & \multirow{3}{*}{$\begin{array}{c}3.14 \\
(9)\end{array}$} & $454(9)$ & 304 & 436 & 2.968 \\
\hline & 110 & & & & $636(13)$ & 430 & 458 & 3.221 \\
\hline & 111 & & & & $259(5)$ & 180 & 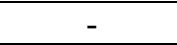 & 2.746 \\
\hline \multirow{3}{*}{$\mathrm{Na}$} & 100 & \multirow{3}{*}{$\operatorname{Im} 3 \mathrm{~m}$} & \multirow{3}{*}{371} & \multirow{3}{*}{$\begin{array}{c}5.7 \\
(13)\end{array}$} & $371(13)$ & 189 & 236 & 2.638 \\
\hline & 110 & & & & $519(18)$ & 267 & 307 & 2.839 \\
\hline & 111 & & & & $212(7)$ & 109 & - & 2.585 \\
\hline \multirow{3}{*}{ K } & 100 & \multirow{3}{*}{$\operatorname{Im} 3 \mathrm{~m}$} & \multirow{3}{*}{337} & \multirow{3}{*}{$\begin{array}{l}10.9 \\
(20)\end{array}$} & $337(20)$ & 124 & 129 & 2.224 \\
\hline & 110 & & & & $472(28)$ & 175.3 & 116 & 2.372 \\
\hline & 111 & & & & $193(11)$ & 71.6 & 112 & 2.18 \\
\hline \multirow{3}{*}{$\mathrm{Rb}$} & 100 & \multirow{3}{*}{$\operatorname{Im} 3 \mathrm{~m}$} & \multirow{3}{*}{312} & \multirow{3}{*}{$\begin{array}{l}13.4 \\
(24)\end{array}$} & $312(24)$ & 101 & 107 & 2.116 \\
\hline & 110 & & & & $437(34)$ & 143 & 92 & 2.243 \\
\hline & 111 & & & & $178(14)$ & 58.4 & 89 & 2.096 \\
\hline \multirow{3}{*}{ Cs } & 100 & \multirow{3}{*}{$\operatorname{Im} 3 \mathrm{~m}$} & \multirow{3}{*}{302} & \multirow{3}{*}{$\begin{array}{l}16.8 \\
(27)\end{array}$} & $302(27)$ & 85 & 92 & 1.974 \\
\hline & 110 & & & & $423(38)$ & 120 & 72 & 2.073 \\
\hline & 111 & & & & $173(15)$ & 49 & 70 & 1.971 \\
\hline
\end{tabular}
surface energy and the work function of the electron 


\section{Description of the empirical model}

In [11], when considering the melting temperature of small particles, we obtained the equation:

$$
\mathrm{T}(\mathrm{r})=\mathrm{T}_{0}\left(1-\frac{\mathrm{R}(\mathrm{I})}{\mathrm{r}}\right) \text {, }
$$

where the parameter $\mathrm{R}(\mathrm{I})$ is determined by the expression:

$$
\mathrm{R}(\mathrm{I})=\frac{2 \sigma \mathrm{U}}{\mathrm{RT}}
$$

where $\sigma$ - surface tension, $v$ - molar volume, R - universal gas constant, $\mathrm{T}$ - temperature.

Experimental studies carried out by us with physical objects on thin films of various nature: mechanical, optical, magnetic, as well as the results of other researchers, have shown the size dependence of all physical properties of small particles and thin films. This dependency looks like this:

$$
\grave{\mathrm{A}}(\mathrm{r})=\grave{\mathrm{A}}_{0}\left(1-\frac{\mathrm{R}(\mathrm{I})}{\mathrm{r}}\right),
$$

where $\mathrm{A}(\mathrm{r})$ is a physical property (mechanical, etc.) that depends on the linear size (dimensional effect), $\mathrm{A}_{0}$ is a physical property (volumetric) that does not depend on size.

It is clear that the surface of a solid is a nanostructure. The same is true for liquid. Equations (7), (8), and (9) have the same structure and diverge as $r \rightarrow 0$; therefore, we will redefine equation (9) and write down finally:

$$
\begin{aligned}
& \grave{\mathrm{A}}(\mathrm{r})=\grave{\mathrm{A}}_{0}\left(1-\frac{\mathrm{R}(\mathrm{I})}{\mathrm{r}}\right), \mathrm{r}>\mathrm{R}(\mathrm{I}), \\
& \mathrm{A}(\mathrm{r})=\mathrm{A}_{0}\left(1-\frac{\mathrm{R}(\mathrm{I})}{\mathrm{R}(\mathrm{I})+\mathrm{r}}\right), \mathrm{R}_{0} \leq \mathrm{r} \leq \mathrm{R}(\mathrm{I}) .
\end{aligned}
$$

This model is shown schematically in Figure 1. It is an ideal atomically smooth single crystal without vacancies, dislocations, and other defects.

The de Broglie layer $\mathrm{R}_{0}=\lambda \mathrm{dB}=\hbar / \mathrm{p}$ for metals ranges from $0.01 \mathrm{~nm}$ to $0.1 \mathrm{~nm}$. Quantum dimensional effects begin in this layer. The main quantum-dimensional structures include structures with a twodimensional electron gas - epitaxial films, MIS structures, heterostructures, etc ; structures with onedimensional gas - quantum threads or wires; structures with a zero-dimensional gas - quantum dots, boxes, crystallites [12].

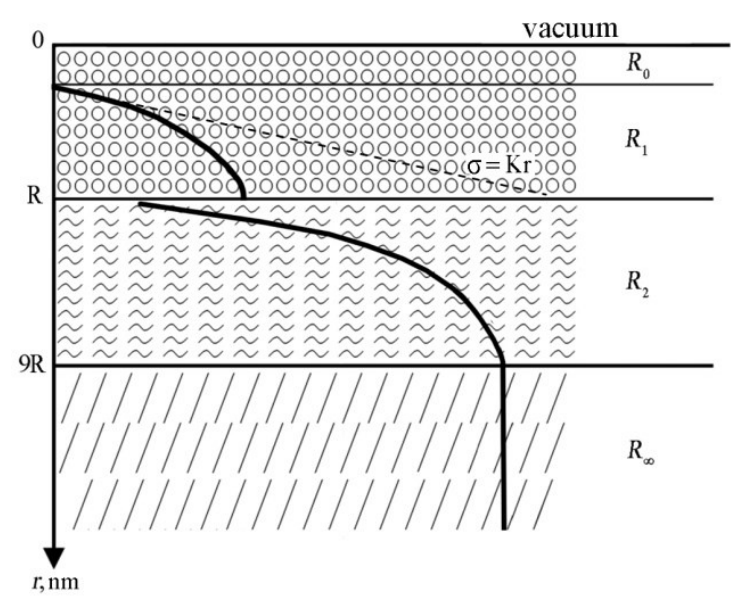

Fig.1. Schematic representation of the surface layer.

Designations: $\mathrm{R}_{0}$ - de Broglie layer; $\mathrm{R}_{1}$ - layer $\mathrm{R}(\mathrm{I}) ; \mathrm{R}_{2}$ is the $\mathrm{R}(\mathrm{II})$ layer; $\mathrm{R}_{\infty}$ - solid sample layer.

The R(I) layer is described by the first dependence from equation (9) (r >> R(I)). In the R(I) layer with pure metal atoms, there is a reconstruction and relaxation associated with the rearrangement of the surface [13]. For gold, the lattice constant is equal to $\mathrm{R}(\mathrm{I})=0.41 \mathrm{~nm}$ and the surface is rearranged at a distance 
$\mathrm{R}(\mathrm{I})_{\mathrm{Au}}=1.2 / 0.41 \approx 3$ of three atomic monolayers. Size effects in the $\mathrm{R}(\mathrm{I})$ layer are determined by the entire group of atoms in the system (collective processes). Such "semiclassical" size effects are observed only in nanoparticles and nanostructures [14]. Experimentally, they can be observed on very pure single crystals with grazing incidence of X-ray radiation, when the angle of incidence is equal to or less than the critical angle of total internal reflection [15]. When the angle of incidence becomes less than the critical one, the refracted wave exponentially decays in the volume at a characteristic depth of the order of several nanometers (for example, this depth is $3.2 \mathrm{~nm}$ for silicon, and $1.2 \mathrm{~nm}$ for gold). As a result, a so-called vanishing wave is formed, which propagates parallel to the surface. Therefore, the diffraction of such waves provides information on the structure of the surface layer [13].

The $\mathrm{R}(\mathrm{II})$ layer extends approximately to the size $\mathrm{R}(\mathrm{II}) \approx 9 \mathrm{R}=\mathrm{R}_{\infty}$, where the bulk phase begins. Dimensional properties begin from this size. By nanomaterials it is customary to understand materials, the main structural elements of which do not exceed the nanotechnological boundary $\sim 100 \mathrm{~nm}$, at least in one direction [14-18]. A number of researchers are of the opinion that the upper limit (maximum size of elements) for nanostructures should be related to some critical characteristic parameter: the mean free path of carriers in transport phenomena, the size of domains/domain walls, the Frank-Read loop diameter for dislocation slip and the like. This means that the R (II) layer should contain many dimensional effects associated with optics, magnetism, and other physical properties according to Eq. (10).

The $\mathrm{R}(\mathrm{II})$ layer is described by the second dependence from equation (10) $\left(\mathrm{R}_{0}<\mathrm{r}<\mathrm{R}(\mathrm{I})\right)$. The parameter $\mathrm{R}(\mathrm{I})$ is related to the surface tension $\sigma$ by formula (7). In [11], it is shown that, with an accuracy of $3 \%$, the relation:

$$
\sigma=10^{-3} \cdot T_{\mathrm{m}},
$$

where $\mathrm{Tm}$ is the melting point of the solid $(\mathrm{K})$. The ratio is fulfilled for all metals and for other crystalline compounds. At $\mathrm{T}=\mathrm{T}_{\mathrm{m}}$, from equation (8) we obtain:

$$
\mathrm{R}(\mathrm{I})_{\mathrm{I}}=0.24 \cdot 10^{-9} \mathrm{v} \text {. }
$$

Equation (12) shows that the thickness of the surface layer R(I) is determined by one fundamental parameter - the molar (atomic) volume of the element $v=M / \rho, M$ is the molar mass $(\mathrm{g} / \mathrm{mol}), \rho$ is the density $\left(\mathrm{g} / \mathrm{cm}^{3}\right)$, which changes periodically in accordance with the table of D.I. Mendeleev. Equation (11) can be empirically rewritten as:

$$
\sigma(\mathrm{hkl})=10^{-3} \cdot T_{\mathrm{m}} \cdot 1(\mathrm{hkl}), .
$$

where 1(hkl) for crystals with body-centered (bcc) and face-centered (fcc) cubic structures is given by relations (6) at $\mathrm{a}=1$.

Let's make calculations according to (12) and (13), taking reference data on $\mathrm{T}_{\mathrm{m}}, \mathrm{M}, \rho$.

\section{Research results and discussion}

Table 2 shows that our empirical model, based on the results of calculations, does not differ much from the method of electron-statistical calculation of the surface energy of metals [3,4] and from the model of coordination melting of crystals $[5,6]$. However, in contrast to these models, our model gives a numerical value for the thickness of the surface layer and, perhaps, this is the first time. The thickness of the surface layer for the $\mathrm{Li} \rightarrow \mathrm{Cs}$ system ranges from $3 \mathrm{~nm}$ to $17 \mathrm{~nm}$. If we recalculate it in lattice constants a, then it turns out that the surface layer contains on average 9 monolayers ( $\mathrm{Li}$ ) to 27 monolayers (Cs). Experimentally, such surface layers can be determined by methods of scattering X-rays during their internal reflection. Using this method, the thickness of the surface layer was determined for gold single crystals (R(I) $=1.2 \mathrm{~nm}$ ) and for silicon $(\mathrm{R}(\mathrm{I})=3.1 \mathrm{~nm})[13]$, that is, this $\mathrm{R}(\mathrm{I})$ layer is a nanostructure ( fig. 1). Let us now consider the system of atoms $\mathrm{Li} \rightarrow$ Cs. Consider a body-centered cubic lattice (BCC) (Fig. 2).

Figure 2 shows that the shift in the crystal occurs most easily along the atomic planes with the densest packing of atoms (Fig. 2, a). Plane ABCD (Fig. 2, b), the number of atoms in the plane ABCD - 1; area $\mathrm{ABCD}=\mathrm{a}^{2}$; area per 1 atom - specific area: $\mathrm{S}=\mathrm{a}^{2} / 1=\mathrm{a}^{2}$ - measure of packing density. Plane ABGH (Fig. $2, \mathrm{c}$ ), the number of atoms in the plane ABGH - 2; area $A B G H=a^{2} \sqrt{2} ; S=a^{2} \sqrt{2} / 2 \approx 0.7 a^{2}<a$.

Since the physical and chemical properties along different directions depend on the density of atoms on them, the listed properties along different directions in crystalline bodies should be unequal (Fig. 3). 


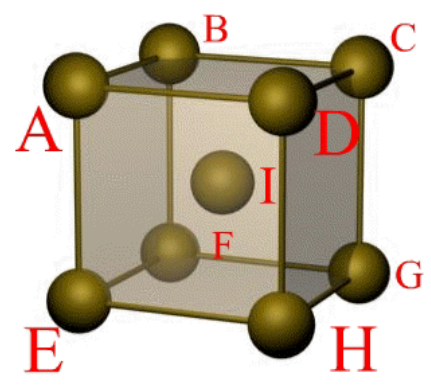

a)

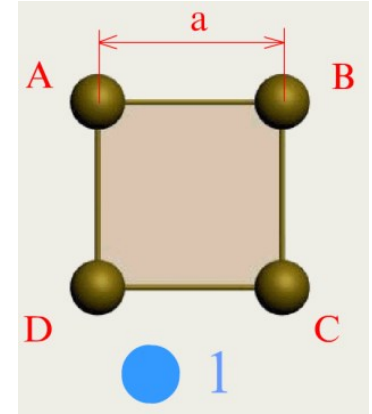

b)

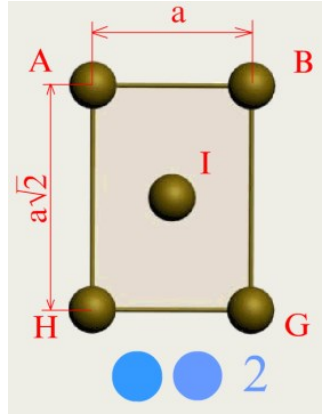

c)

Fig.2. Volume-centric cubic lattice: a) unit cell of the bcc lattice; b) plane ABCD. The number of atoms in the plane $\mathrm{ABCD}-1$; c) plane ABGH. Number of atoms in the plane ABGH - 2
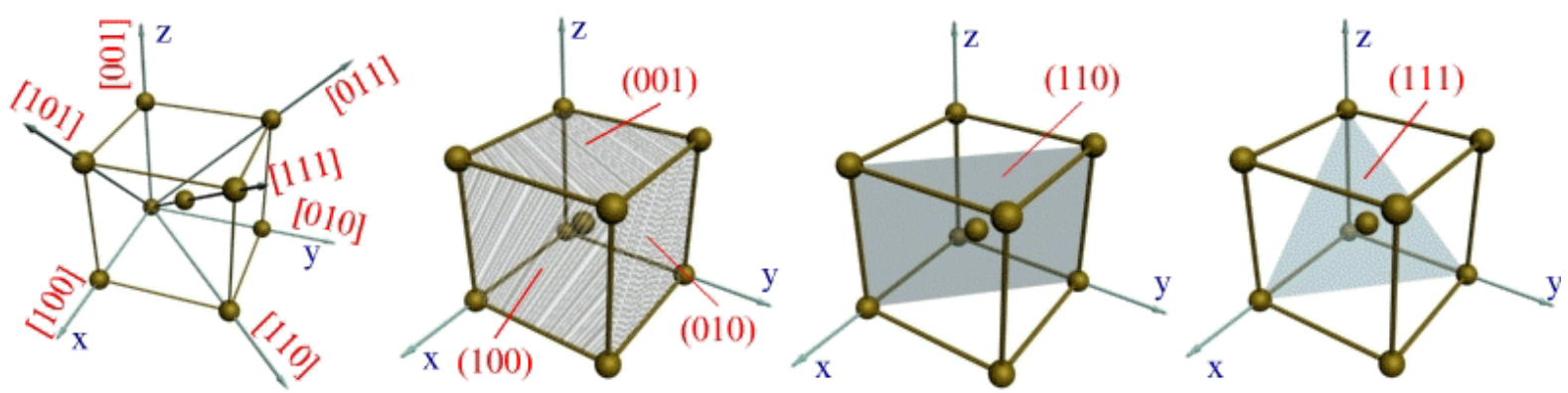

Fig.3. Crystallographic directions and planes in the crystal lattice: a) - the main directions and their designation; b), c), d) -basic planes and their designation

Table 2 shows that the body-centered cubic lattice $\mathrm{Li} \rightarrow$ Cs in the (110) plane contains more atomic monolayers, and the (111) layer contains the smallest number of atomic monolayers. These layers are located in the R(I) layer (Fig. 1), where the relaxation or reconstruction of the single crystal surface occurs (Fig. 4) [13].
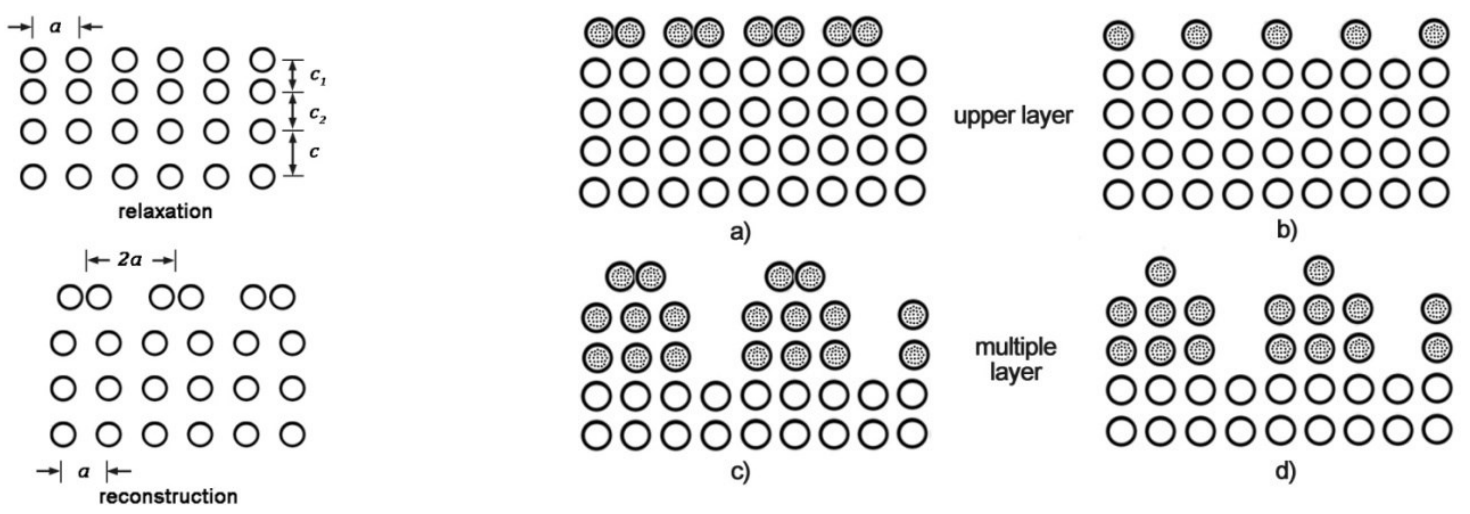

Fig.4. Transformation of the metal surface:

relaxation - upper layer $(\mathrm{a}, \mathrm{b})$; reconstruction - several layers $(\mathrm{c}, \mathrm{d})$

The relaxation of the surface is understood as the difference between the distances between the last crystallographic planes parallel to the plane of the interface with vacuum from the distances between the same planes in the volume (Fig. 4). The variety of cases when the atomic structure of the upper layer is modified is characterized by the term reconstruction (it is especially relevant for semiconductors). Relaxation is characteristic of metals. The same is expected for $\mathrm{Li} \rightarrow$ Cs crystals, and from Table 2 it can be seen that the surface layer extends over several atomic monolayers (Fig. 4, right). On the whole, size effects are observed up to distances $\mathrm{R}(\mathrm{II}) \approx 9 \mathrm{R}=\mathrm{R}_{\infty}$, where the bulk phase begins (Fig. 1), which, starting with potassium, exceeds the limit of $100 \mathrm{~nm}$, which is characteristic of Slater nanostructures [19]. Table 2 shows 
that the work function of electrons changes proportionally with a change in the surface energy of the metal. This means that the device developed by us $[1,2]$ can be used to measure the state of the metal surface and its anisotropy.

\section{Conclusion}

The anisotropy of the surface of its metals, even of atomically smooth surfaces, began to be developed relatively recently. First, this is due to an incomplete understanding of the role that surface energy and its anisotropy play in nanostructures. Secondly, because of the difficulty of measuring it. Our proposed model gives a numerical value for the thickness of the surface layer and allows one to evaluate the physical processes that occur in nanostructures. The de Broglie layer $\mathrm{R}_{0}=\lambda \mathrm{dB}=\hbar / \mathrm{p}$ for metals ranges from $0.01 \mathrm{~nm}$ to $0.1 \mathrm{~nm}$. Quantum dimensional effects begin in this layer. The main quantum-dimensional structures include structures with a two-dimensional electron gas - epitaxial films, MIS structures, heterostructures, etc.; structures with one-dimensional gas - quantum threads or wires; structures with zero-dimensional gas quantum dots, boxes, crystallites. The R(I) layer is described by the first dependence from equation (9) ( $\mathrm{r}>>$ $\mathrm{R}(\mathrm{I})$ ). In the $\mathrm{R}(\mathrm{I})$ layer with pure metal atoms, there is a reconstruction and relaxation associated with the rearrangement of the surface. The $\mathrm{R}$ (II) layer extends approximately to the size $\mathrm{R}(\mathrm{II}) \approx 9 \mathrm{R}=\mathrm{R}_{\infty}$, where the bulk phase begins. Dimensional properties begin from this size. We have shown for the first time in the world that the thickness of the surface layer is determined by one fundamental parameter - the molar (atomic) volume of an element, which periodically changes in accordance with the table of D.I. Mendeleev.

\section{Acknowledgments}

\section{The work was carried out under the program of the Ministry of Education and Science of the Republic of} Kazakhstan Grant No. 0118RK000063.

\section{REFERENCES}

1 Yurov V.M. Oleshko V.S. The impact of the environment on the contact potential difference of metal machine parts. Eurasian Physical Technical Journal, 2019. Vol.16, No.1(31), pp. $99-108$.

2 Yurov V.M., Makeeva O.V., Oleshko V.S., Fedorov A.V. Development of a device for determining work electron output. Eurasian Physical Technical Journal, 2020, Vol.17, No.1 (33), pp. 127 - 131.

3 Shebzukhova I. G., Arefieva L. P. Anisotropy of surface energy and the electron work function IIB of metals. Journal of technical physics, 2019, Vol. 89, Issue. 2, pp. $306-309$.

4 Shebzukhova I. G., Arefieva L. P. Estimation of polarization and dispersion corrections to the surface energy of the faces of metal crystals. Physicochemical aspects of studying clusters, nanostructures and nanomaterials, 2020, Issue 12 , pp. $319-325$

5 Bokarev V.P., Krasnikov G.Ya. Anisotropy of physicochemical properties of single-crystal surfaces. Electronic technology. Series 3. Microelectronics, 2016, No. 4(164), pp. 25 - 30.

6 Bochkarev V.P. Development of physical and chemical principles for assessing the effect of surface energy on the properties of materials and processes for microelectronic technology. Doctor Dissert. of Techn. Scinc. degree, Moscow, 2020. - $299 \mathrm{p}$.

7 Bernstein M.L., Rakhshtadt A.G. Element properties. Part 1. Physical properties. Moscow, 1969, 600 p.

8 Jian Wang, Shao-Qing Wang. Surface energy and work function of FCC and BCC crystals: Density functional study. Surface Science, 2014, Vol. 630, pp. 216-224.

9 Vladimirov A.F. Anisotropy of the work function of the electron and reticular compaction of "loose" faces of metal crystals. Surface, X-ray, synchrotron and neutron research, 1999, No. 9, pp. 58 - 66.

10 Skriver H.L., Rosengaard N.M. Surface energy and work function of elemental metals. Physical Reviev B, 1992, Vol. 46, No. 11, pp. $7157-7168$.

11 Yurov V.M., Guchenko S.A., Laurinas V.Ch. Surface layer thickness, surface energy, and atomic volume of an element. Physicochemical aspects of studying clusters, nanostructures and nanomaterials, 2018, Vol. 10, pp. 691-699.

12 Arutyunov K.Yu. Quantum size effects in metallic nanostructures. RAS reports. 2015. No. 3(28), pp. 7 - 16.

13 Oura K., Lifshits V.G., Saranin A.A., t al. Katayama M. Introduction to surface physics. Moscow, 2006,490 p.

14 Uvarov N.F., Boldyrev V.V. Size effects in the chemistry of heterogeneous systems. Uspekhi khimii, 2001, Vol. 70(4), pp. 307 - 329. [in Russian]

15 Gusev A.I. Nanomaterials, nanostructures, nanotechnology. Moscow, 2005, 412 p. [in Russian]

16 Andrievsky R.A., Ragulya A.V. Nanostructured materials. Moscow, 2005, 192 p. [in Russian]

17 Suzdalev I.P. Nanotechnology: physical chemistry of nanoclusters, nanostructures and nanomaterials. Moscow, 2006, 592 p. [in Russian]

18 Kiselev V.F., Kozlov S.N., Zoteev A.V. Fundamentals of Solid Surface Physics. Moscow, 1999, 284 p.

19 Gleiter H. Nanostructured materials: basic concepts and microstructure. Acta mater. 2000, Vol. 48, pp. 1 - 29. 